\title{
Qualidade de vida dos pacientes hipertensos em uma unidade de saúde
}

\author{
Life quality of hypertensive patients in a health care unit

\section{La calidad de vida de los pacientes hipertensos en un centro de atención médica}

André Alexey Polidoro. Pontifícia Universidade Católica do Paraná (PUCPR). Curitiba, PR, Brasil. ande_alexey@hotmail.com (Autor correspondente)

Marcelo Garcia Kolling. Pontifícia Universidade Católica do Paraná (PUCPR). Curitiba, PR, Brasil. garciakolling@gmail.com

\section{Resumo}

Objetivo: Avaliar a autopercepção da qualidade de vida dos pacientes hipertensos. Métodos: Foi realizado um estudo quantitativo transversal, por meio da aplicação do questionário o SF-36 (Medical Outcomes Study 36-Item Short-Form Health Survey) para 47 pacientes hipertensos de uma Unidade Municipal de Saúde do Município de Curitiba, sendo 10 homens e 37 mulheres. Resultados: Os resultados mostraram que a média global de saúde da população foi 61,0, variando do escore 11,0 até 92,1. O critério melhor avaliado foi "limitação por aspectos sociais", enquanto o pior aspecto foi "limitação pela dor". Conclusão: Os resultados apontam para a necessidade de não se desprezar nenhum dos componentes da qualidade de vida no cuidado aos pacientes hipertensos, com risco de causar prejuízo na mesma.

\section{Abstract}

Objective: To assess the self-perceived life quality of hypertensive patients. Methods: It was carried out a quantitative and cross-sectional study by applying the questionnaire SF-36 (Medical Outcomes Study 36-Item Short-Form Health Survey) to 47 hypertensive patients of a health care unit at Curitiba, being 10 men and 37 women. Results: The results showed that the overall average health of the population was 61.0 , ranging from 11.0 to 92.1 score. The best rated criterion was "limited by social", while the worst aspect was "limited by pain". Conclusion: The results indicate the need of not neglecting any component of life quality in the care of hypertensive patients, with the risk of impairing it.

\section{Resumen}

Objetivo: Evaluar la percepción subjetiva de la calidad de vida de los pacientes hipertensos. Métodos: Un estudio cuantitativo de corte transversal se llevó a cabo mediante la aplicación del cuestionario SF-36 (Medical Outcomes Study 36-Tema Short-Form Health Survey) a 47 pacientes hipertensos de una Unidad Municipal de la ciudad de Curitiba, siendo 10 hombres y 37 mujeres. Resultados: Los resultados mostraron que el promedio general de salud de la población era de 61,0 , que iba desde 11,0 hasta 92,1 de puntuación. El criterio de mayor rating fue "limitado por el social", mientras que el peor aspecto era "limitado por el dolor". Conclusión: Los resultados indican la necesidad de no descuidar ningún componente de la calidad de vida de los componentes de la atención de los pacientes hipertensos con riesgo de causar daños en el mismo.

\section{Palavras-chave:}

Qualidade de Vida

Hipertensão

Inquéritos e Questionários

Avaliação de Resultados

(Cuidados de Saúde)

Keywords:

Quality of Life

Hypertension

Surveys and Questionnaires

Outcome Assessment

(Health Care)

\section{Palabras clave:}

Calidad de Vida

Hipertensión

Encuestas Y Cuestionarios

Evaluación de de Resultado

(Atención de Salud)
Como citar: Polidoro AA, Kolling MG. Qualidade de vida dos pacientes hipertensos em uma unidade de saúde. Rev Bras Med Fam Comunidade. 2016;11(38):1-7. http://dx.doi.org/10.5712/rbmfc11(38)976
Fonte de financiamento: declaram não haver.

Parecer CEP: 287.069 (PUCPR), aprovado em 28/05/2013

Conflito de interesses: declaram não haver. Procedência e revisão por pares: revisado por pares.

Recebido em: 18/07/2014.

Aprovado em: 15/02/2016. 


\section{Introdução}

A hipertensão arterial sistêmica (HAS) é uma importante causa de morbimortalidade,$^{1} \mathrm{com}$ mais de 7 milhões de óbitos no mundo e inúmeras complicações. O Brasil tem evoluído muito na detecção e controle da hipertensão arterial à medida que aumenta a cobertura da Estratégia Saúde da Família. ${ }^{2}$

Entretanto, seria reducionista e incorreto analisar a abordagem destes pacientes somente com as taxas de pressão arterial, embora fundamentais no planejamento em saúde ${ }^{3}$ desconsiderando a 'importância crucial das emoções', ${ }^{4}$ especialmente por se tratar de uma patologia muito ligada ao estilo de vida. ${ }^{5}$

Mas, inclusive para o planejamento, não se pode entender a saúde do paciente hipertenso em uma relação exclusiva com os valores da pressão arterial, afinal "a saúde é um conceito positivo que enfatiza recursos sociais e pessoais". ${ }^{6} \mathrm{Um}$ olhar com esse viés pode fazer com que as ações desenvolvidas na Atenção Primária à Saúde sejam muito seletivas e direcionadas para um único indicador, em detrimento de outros aspectos muito importantes para a saúde das pessoas. ${ }^{7}$

Por esse motivo, novas pesquisas estão sendo feitas a respeito da qualidade de vida desses indivíduos, que, segundo a Organização Mundial da Saúde (OMS), "é a percepção do indivíduo sobre sua posição na vida, considerada no contexto da cultura e dos valores nos quais vive e elabora seus objetivos, expectativas, padrões e preocupações". ${ }^{8}$

A avaliação da qualidade de vida não é uma tarefa fácil. O próprio conceito de qualidade de vida pode somente ser observado subjetivamente, pela própria pessoa, sendo a abordagem "de dentro para fora". ${ }^{9}$ Conforme aprendemos na psicologia, "a realidade é a de cada um". ${ }^{10}$

Para avaliar a autopercepção da qualidade de vida, uma ferramenta comumente utilizada é o Medical Outcomes Study 36-Item Short-Form Health Survey (SF-36), um questionário autoaplicável já validado internacionalmente, e que estabelece valores para cada resposta subjetiva. ${ }^{11} \mathrm{E}$ uma ferramenta importante na tentativa de obter respostas objetivas, possíveis de comparação entre tempos e locais diferentes, e, ao mesmo tempo, dirigir nosso foco ao bem-estar do indivíduo de quem cuidamos.

Portanto, se por um lado, com o uso do SF-36, perdem-se a riqueza da subjetividade, da narrativa, da cultura e do acesso à cosmovisão do paciente, por outro lado, o questionário objetivo permite comparação de resultados em diferentes locais, ou em diferentes momentos, facilitando ensaios clínicos e econômicos, até mesmo para teste avaliação de resultados e impactos após implantação de alguma medida. ${ }^{12}$

Mas, sua finalidade maior é analisar a compreensão integral do indivíduo sobre sua saúde, no intuito de ajudá-lo a tomar decisões sobre seu tratamento. ${ }^{11}$ Já reconhecida internacionalmente, essa ferramenta foi validada em português e foi objeto de adaptação cultural. ${ }^{13}$

O objetivo desta pesquisa é avaliar a autopercepção da qualidade de vida dos pacientes inscritos no programa de hipertensão de uma Unidade de Saúde no Município de Curitiba, PR. O objetivo secundário é descobrir como são avaliados cada um dos componentes da qualidade de vida, conforme o SF-36.

\section{Métodos}

Trata-se de uma pesquisa quantitativa, descritiva, observacional, não experimental, realizada por meio de um estudo com coleta de dados transversal, em ambiente natural. Como critérios de inclusão 
na pesquisa, exigiu-se que fossem maiores de idade, com diagnóstico de hipertensão arterial sistêmica, cadastrados há pelo menos um ano no Programa de Controle de Hipertensão do município de Curitiba, que concordassem em participar voluntariamente e fossem capazes de responder ao questionário. Como critérios de exclusão, não estar de acordo com um ou mais pontos dos critérios de inclusão. Quarenta e sete pessoas responderam ao questionário.

A coleta de dados foi realizada no mês de setembro de 2013, pelos próprios pesquisadores. Os pacientes foram sorteados manualmente a partir de lista de pacientes do Programa de Hipertensos da área, conforme lista cedida pela Secretaria Municipal da Saúde. Foi feita uma randomização das ruas do bairro, sorteando-se seis ruas para facilitar a coleta de dados, sendo este sorteio realizado manualmente, com a impressão dos nomes das ruas em papéis, colocados em uma caixa e retirados por um terceiro, que não participou em nenhum outro momento da pesquisa. Assim, os dados foram coletados com os pacientes, conforme a ordem numérica crescente de casas nestas ruas e encerrando a pesquisa após obter 47 entrevistas que preenchiam os critérios de inclusão. Houve apenas uma recusa.

Todos os participantes assinaram Termo de Consentimento Livre e Esclarecido e o projeto foi previamente aprovado no Comitê de Ética para Pesquisas em Seres Humanos da Pontifícia Universidade Católica (PUC) do Paraná quanto à viabilidade e no Comitê de Ética da Secretaria Municipal de Saúde de Curitiba para a concessão do campo de estágio.

Aos pacientes foi aplicado o questionário SF-36, além de colhidos dados pessoais (gênero, idade e renda familiar). O SF-36 é um questionário multidimensional formado por 36 itens, englobados em 8 escalas ou componentes: capacidade funcional (10 itens), limitação por aspectos físicos (4 itens), limitação pela dor (2 itens), estado geral de saúde (5 itens), vitalidade (4 itens), aspectos sociais (2 itens), aspectos emocionais (3 itens), saúde mental (5 itens) e mais uma questão de avaliação comparativa entre as condições de saúde atual e de um ano atrás. ${ }^{13}$

Este último item não foi registrado neste estudo por não fazer parte do cálculo do escore total e por fugir dos objetivos do mesmo. Para chegar a um resultado objetivo, cada um desses componentes foi transformado em uma escala que vai de zero a cem pontos, gerando o escore pela interpretação própria da escala. Quanto maior o escore, melhor a qualidade de vida indicada. A média de todos os componentes gera uma nota global de saúde.

A UMS Sambaqui foi escolhida por ter sua organização de trabalho baseada na "Estratégia de Saúde da Família" (ESF) desde sua implantação, sendo que a área 2 (com 336 pacientes hipertensos) é atendida pelos residentes de Medicina de Família e Comunidade (MFC), da PUC. Localiza-se na periferia e ao sul da cidade de Curitiba, capital do Paraná. Os pesquisadores não possuiam, no momento da pesquisa, envolvimento com as atividades assistenciais da unidade de saúde.

\section{Resultados}

Participaram do estudo 47 indivíduos, sendo 10 homens e 37 mulheres, distribuídos conforme a Tabela 1.

Observamos que a idade dos participantes variou entre 31 e 93 anos, sendo que $50 \%$ da população participante tem idade acima de 60 anos (mediana igual a 60). 
Tabela 1. Distribuição dos indivíduos pesquisados por idade e gênero.

\begin{tabular}{lccc} 
& Homens & Mulheres & Total \\
\hline 30 - 39 anos & & 2 & 2 \\
$40-49$ anos & 2 & 5 & 7 \\
50 - 59 anos & 2 & 11 & 13 \\
60 - 69 anos & 1 & 11 & 12 \\
70 anos ou mais & 5 & 7 & 12 \\
não sabe & & 1 & 1 \\
total & 10 & 37 & 47 \\
\hline
\end{tabular}

A qualidade de vida, obervada pelo questionário SF-36, apresentou escore bruto mínimo de 11,0 e máximo de 92,1 , sendo que $50 \%$ da amostra apresentou escore bruto acima de 63,0. A média global de saúde da população foi 61,0 .

Dentre os aspectos considerados no já citado questionário, os critérios melhor avaliados foram "limitação por aspectos sociais" $(80,0)$ e "vitalidade" $(79,0)$, enquanto o pior aspecto foi "limitação pela dor" $(49,2)$ (Figura 1).

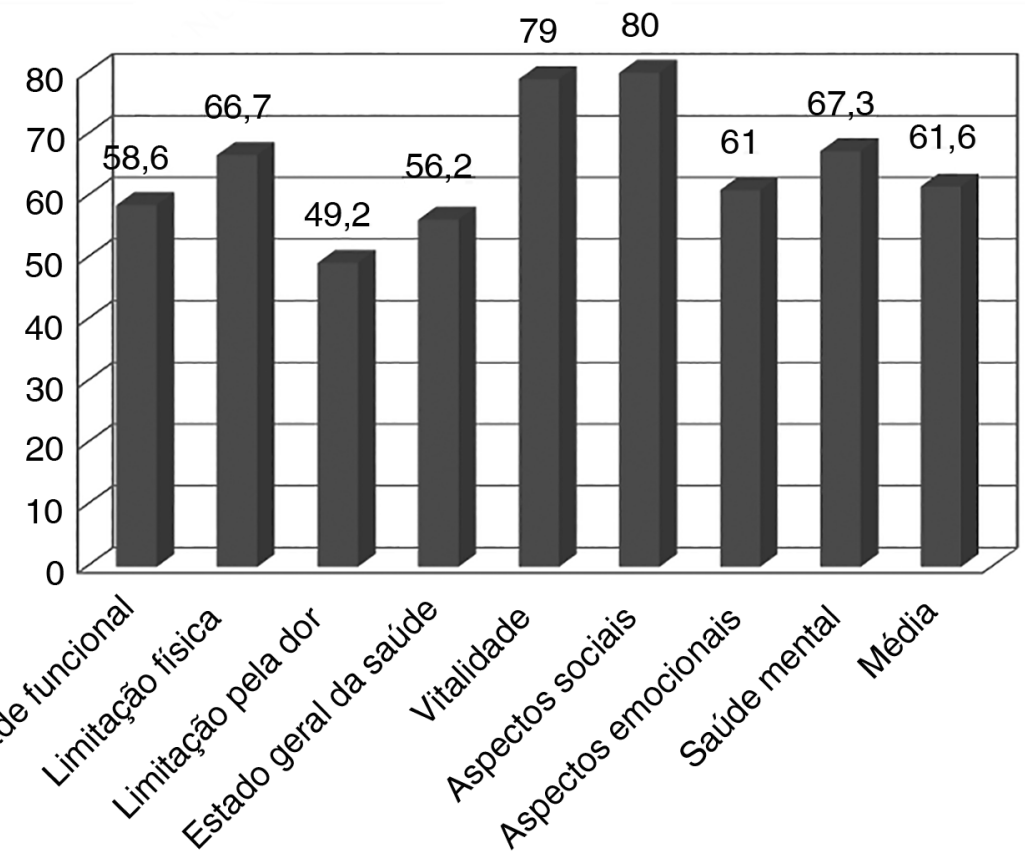

Figura 1. Média dos escores alcançados pelos pacientes no SF-36, por componente.

\section{Discussão}

Os resultados da pesquisa mostram variação importante na qualidade de vida entre os entrevistados, com escore bruto mínimo de 11,0 e máximo de 92,1. Este dado indica que há muitos fatores associados à qualidade de vida de cada pessoa e também ao modo como a mesma entende sua saúde. Isso impede que cometamos o erro de esboçar qualquer associação reducionista. Este estudo diferencia-se de vários outros com o mesmo tema por não excluir pacientes com outras comorbidades, já que estes são a maioria dos pacientes atendidos na APS. 
Mesmo assim, em uma população em que metade dos entrevistados tem mais de 60 anos e em que todos possuem pelo menos um fator de risco cardiovascular (hipertensão arterial) é de destaque positivo a descoberta de que $50 \%$ das pessoas possuem escore bruto maior que 63,0 , com média de 61,0 . Além disso, a média oculta uma grande variação entre os escores individuais. Esta é uma questão interessante e se relaciona ao que já está relatado na literatura como diferenciação entre a doença propriamente dita, disease, e a experiência do indivíduo com a doença, illness. ${ }^{14}$

Dentre as médias de escore bruto de todos os pacientes no mesmo questionário, o critério melhor avaliado em números absolutos foi "aspectos sociais" $(80,0)$, enquanto o pior aspecto foi a "limitação pela dor" $(49,2)$, conforme Figura 1. Este resultado assemelha-se com pesquisa realizada em Fortaleza, com amostra maior, o que mostra a validade externa do teste, ${ }^{15}$ sendo que, naquele, porém, a limitação pela dor teve índice melhor que estado geral de saúde.

Um estudo com 256 pacientes, realizado em Minas Gerais, mostrou nota maior em todos os critérios, mas em sua metodologia, excluía pacientes obesos, pacientes com lesões de órgãos-alvo e pacientes com outras comorbidades, diferenciando-se do objetivo deste estudo. Mesmo assim, os aspectos sociais ainda tiveram a maior média. ${ }^{16}$ Em estudo realizado em Presidente Prudente-SP, com 100 pacientes, houve concordância com o escore bruto maior do critério "aspectos sociais". Também neste, a dor não obteve o menor escore bruto, podendo haver associação com o fato de ter sido feito em um ambulatório de especialidades. ${ }^{17}$

Vale destacar que a dor, aspecto com pior escore neste estudo, possui tratamento, em sua maior parte, disponível no Sistema Único de Saúde e na Atenção Básica.

No caso da população estudada, os resultados de escore bruto médio e escore por componente permitem à equipe de saúde da família o planejamento das açõese a verificação dos resultados destas. As avaliações individuais permitem individualizar a abordagem, percebendo, a priori, temas que podem trazer mais benefício à qualidade de vida do mesmo, auxiliando no "tempo de preparação antes da consulta". ${ }^{18}$

\section{Conclusão}

No presente estudo, concluimos que a população de hipertensos da área 2 da unidade de saúde estudada apresenta um escore bruto médio de 61,0, conforme o questionário SF-36, porém com variação na resposta individual de 11,0 a 92,1. Foi percebido ainda que, entre os componentes desta escala, o pior desempenho de "limitação pela dor" (escore de 49,2). O componente com melhor desempenho foi "aspectos sociais" (escore de 80,0). Outros componentes estão descritos na Figura 1. Concluímos também que a escala SF-36 foi eficiente ao observar a autopercepção das pessoas sobre a qualidade de vida.

Observa-se, também, que o SF-36 não permite conclusões acerca da hipertensão em si, pois se trata de um questionário genérico de qualidade de vida e, por isso, produzindo resultados relacionados à pessoa como um todo, permitindo que o resultado seja influenciado por várias patologias, condições de vida e subjetividade. Este questionário pode ser usado associado a outros instrumentos ou à pesquisa qualitativa a depender do questionamento da pesquisa.

Conclui-se, ainda, a necessidade de mais estudos sobre este tema tão rico, vasto e multifacetado, que observem fatores que influenciam a qualidade de vida dos pacientes, e apontem para modos de como obter este impacto positivo, para que a medicina se aproxime da necessidade de "tratar das pessoas e não das doenças". ${ }^{19}$ 
O controle pressórico dos pacientes hipertensos é sobremodo importante para a prevenção de desfechos negativos relacionados a este fator de risco que, indubitavelmente, afetam a qualidade de vida. Ao mesmo tempo, o cuidado integral ao paciente hipertenso permite abordagens que elevem a sua qualidade de vida e esta não está relacionada apenas à prevenção de longo prazo, mas também a tratamento de diversas outras questões e à promoção da saúde.

Há muito tempo, conhecer o paciente na sua integralidade é prerrogativa da Medicina de Família e Comunidade e isto é importante em todos os seus campos de atuação. Foi percebido, nesta pesquisa, que o fator mais envolvido na piora da qualidade de vida foi limitação pela dor. Os recursos para alívio desse sofrimento estão presentes na unidade de saúde, e podem ser mobilizados tanto no alívio da dor física quanto da dor com origem emocional. Esta simples medida é capaz de melhorar a qualidade de vida do indivíduo como um todo, e não pode ser ignorada no atendimento daquele indivíduo.

Ações programáticas que se foquem demasiadamente nos parâmetros biomédicos, corroborados por agendas que são construídas a partir de uma condição específica de saúde (tarde do hipertenso, por exemplo) podem fazer com que não haja espaço para o atendimento ou para o surgimento da queixa de dor, que tem limitado a qualidade de vida destes pacientes. O fato citado diminui a qualidade da atenção primária. ${ }^{20}$

Além disso, a individualização do paciente é um dos pilares da prática do médico de família e comunidade. Esta especialidade é comprometida sempre, e em primeiro lugar, com o indivíduo. Não se trata de órgãos, aparelhos, ou grupo de doenças. Não se discrimina gênero ou idade ${ }^{21}$ e dedica-se, inclusive, a indivíduos saudáveis. ${ }^{4}$ Faz-se mandatório avaliar os sentimentos e as emoções do indivíduo em relação ao seu problema, avaliando preocupações e impacto na qualidade de vida. ${ }^{18} \mathrm{~A}$ visão reducionista é antagônica à integralidade e provém da "incapacidade de estabelecer uma relação com o outro a não ser transformando-o em objeto". ${ }^{22}$

Da mesma maneira, o cuidado com a qualidade de vida não deve invalidar o princípio de que as ações precisam ter impactos epidemiológicos positivos, mas ir além, ou seja, saber que a melhora epidemiológica não é o único objetivo dos serviços de saúde. ${ }^{23}$

\section{Referências}

1. Williams B. The year in hypertension. J Am Coll Cardiol. 2009;55(1):65-73. PMID: 20117366 DOI: http://dx.doi.org/10.1016/j. jacc.2009.08.037

2. Rosário TM, Scala LCNS, França GVA, Pereira MRG, Jardim PCBV. Prevalência, controle e tratamento da hipertensão arterial sistêmica em Nobres, MT. Arq Bras Cardiol. 2009;93(6):672-8.

3. Rouquayrol MZ, Almeida Filho N. Epidemiologia \& Saúde. 6a ed. Rio de Janeiro: Guanabara Koogan; 2003. 708p.

4. McWhinney IR, Freeman T. Textbook of Family Medicine. New York: Oxford University Press; 2009. 472p.

5. Lessa I. Introdução à epidemiologia das doenças cardiovasculares no Brasil. In: Lessa I. O adulto brasileiro e as doenças da modernidade. Epidemiologia das doenças crônicas não-transmissíveis. São Paulo: Hucitec; 1998;73-6.

6. World Health Organization. Milestones in health promotion Statements from global conferences. Geneva: World Health Organization; 2009.

7. Organização Mundial da Saúde. Classificação Internacional de Funcionalidade, Incapacidade e Saúde [Centro Colaborador da Organização Mundial da Saúde para a Família de Classificações Internacionais, org. coordenação da tradução, Buchalla CM. São Paulo: Editora da Universidade de São Paulo: EDUSP; 2003. 
8. WHOQOL Group. Development of the WHOQOL: Rationale and current status. Int J Ment Health. 1994;23(3):24-56. DOI: http://dx.doi.org/10.1080/00207411.1994.11449286

9. Segre M, Ferraz FC. O conceito de saúde. Rev Saúde Pública. 1997;31(5):537-42.

10. Bion WR. Uma teoria sobre o processo de pensar. In: Estudos psicanalíticos revisados (Second Thoughts). Rio de Janeiro: Imago; 1967. p.101-9.

11. Ware JE Jr, Sherbourne CD. The MOS 36-item short-form health survey (SF-36). I. Conceptual framework and item selection. Med Care. 1992;30(6):473-83.

12. Bowling A, Brazier J. Quality of life in social science and medicine. Soc Sci Med. 1995;41(10):1337-8. DOI: http://dx.doi. org/10.1016/0277-9536(95)00148-Z

13. Ciconelli RM. Tradução para o português e validação do questionário genérico de avaliação de qualidade de vida "Medical Outcomes Study 36-item Short-form Health Survey (SF-36)" [Tese de Doutorado]. São Paulo: Universidade Federal de São Paulo; 1997.

14. Gusso G, Poli Neto P. Gestão da Clínica. In: Gusso G, Lopes JMC. Tratado de medicina de família e comunidade: princípios, formação e prática. Porto Alegre: Artmed; 2012. p.159-66.

15. Brito DMS, Araújo TL, Galvão MTG, Moreira TMM, Lopes MVO. Qualidade de vida e percepção da doença entre portadores de hipertensão arterial. Cad Saúde Pública. 2008;24(4):933-40. DOl: http://dx.doi.org/10.1590/S0102-311X2008000400025

16. Silqueira SMF. O questionário genérico SF-36 como instrumento de mensuração da qualidade de vida relacionada a saúde de pacientes hipertensos [Tese de doutorado]. Escola de Enfermagem de Ribeirão Preto, USP-RP. Recuperado em 201402-05. [Acesso 2 Jan 2014]. Disponível em: http://www.teses.usp.br/teses/disponiveis/22/22133/tde-17052007-160822/

17. Cavalcante MA, Bombig MTN, Luna Filho B, Carvalho ACC, Paola AAV, Povoa R. Qualidade de vida de pacientes hipertensos em tratamento ambulatorial. Arq Bras Cardiol. 2007;89(4):245-50. DOI: http://dx.doi.org/10.1590/S0066782X2007001600006

18. Ramos V. A consulta em 7 passos - Execução e análise crítica de consultas em medicina geral e familiar. Lisboa: VFBM Comunicação; 2008.

19. Roncoleta AFT, Moreto G, Levites MR, Janaudis MA, Blasco PG, Leoto RF. Princípios da Medicina de Família. São Paulo: Sobramfa; 2003.

20. Fabrega H. Disease and social behavior. An interdisciplinary perspective. Cambridge: MIT Press; 1974.

21. Pendleton D, Schofield T, Tate P, Havelock P. The new consultation: developing doctor-pacient communication. Oxford: Oxford University Press; 2003.

22. Santos BS. A crítica da razão indolente: contra o desperdício da experiência. São Paulo: Cortez; 2000.

23. Mattos RA. Os sentidos da integralidade: algumas reflexões acerca dos valores que merecem ser defendidos. In: Pinheiro $R$, Mattos RA, orgs. Os sentidos da integralidade na atenção e no cuidado à saúde. Rio de Janeiro: IMS- UERJ/ABRASCO; 2001. p.39-64. 